\title{
Local Evaluation of Processed Membrane Electrode Assemblies by Scanning Electrochemical Microscopy
}

\author{
Wiebke Schulte, ${ }^{\text {a }}$ Shuai Liu, ${ }^{\mathrm{b}}$ Inka Plettenberg, ${ }^{\text {a }}$ Susanne Kuhri, ${ }^{\text {b }}$ Wiebke Lüke, ${ }^{\mathrm{b}}$ \\ Werner Lehnert, ${ }^{\mathrm{b}, \mathrm{c}, \mathrm{z}}$ and Gunther Wittstock ${ }^{\mathrm{a}, *, \mathrm{z}}$
}

\author{
${ }^{a}$ School of Mathematics and Natural Sciences, Institute of Chemistry, Carl von Ossietzky University of Oldenburg, \\ $D$-26129 Oldenburg, Germany \\ ${ }^{b}$ Institute of Energy and Climate Research, Electrochemical Process Engineering (IEK-3), Forschungszentrum Jülich \\ GmbH, D-52425 Jülich, Germany \\ ${ }^{c}$ Faculty of Mechanical Engineering, RWTH Aachen University, Aachen, Germany
}

\begin{abstract}
Gas diffusion electrodes (GDEs) for high-temperature polymer electrolyte fuel cells with different sizes of the used binder particles were evaluated by scanning electrochemical microscopy (SECM) with shear force (SF) supplement. The SF data provide means of checking the substrate morphology with respect to cracks formed during the drying process and with respect to aggregates from used binder of poly(fluoroethylene) (PTFE) simultaneously to the electrochemical data. Electron microscopy results show that a GDE prepared with smaller PTFE particles exhibits less PTFE aggregation and more regular cracks. The SECM images show a more homogeneous distribution and higher level of oxygen reduction reaction activity for the GDE prepared with smaller PTFE particles. The quantitative comparison is enabled by the SF setup that maintains a constant working distance toward the sample in the variant of the redox competition mode, in which a cyclic voltammogram was recorded for every grid position of the microelectrode probe. Mass transport limitations of oxygen during the experiment are avoided by dedicated shape of the microelectrode body. Images of microelectrode currents at specific potentials were extracted to map the local electrocatalytic activity of the GDE. The GDEs were processed to membrane electrode assemblies and applied in HT-PEFC single cell tests. The polarization curve agree with the SECM results that GDEs produced with smaller PTFE particles favor the MEA performance.

(C) The Author(s) 2017. Published by ECS. This is an open access article distributed under the terms of the Creative Commons Attribution 4.0 License (CC BY, http://creativecommons.org/licenses/by/4.0/), which permits unrestricted reuse of the work in any medium, provided the original work is properly cited. [DOI: 10.1149/2.0061709jes] All rights reserved.

(cc) BY
\end{abstract}

Manuscript submitted April 12, 2017; revised manuscript received June 2, 2017. Published June 14, 2017.

The concept of zero-emission requires a rapid shift from traditional fuels to next generation of clean technologies ${ }^{1}$ to mitigate local pollution in urban areas and to mitigate the emission of carbon dioxide. Polymer electrolyte water electrolyzers for the hydrogen production (from solar and wind power) and polymer electrolyte fuel cells (PEFC) for electricity production from hydrogen are considered one of the most promising clean power technologies. Both electrochemical components are equipped with membrane electrode assemblies (MEAs) consisting either of gas diffusion electrodes (GDEs) assembled with membranes or catalyst coated membranes (CCMs) combined with gas diffusion layers (GDLs). ${ }^{2,3}$ In the high-temperature polymer electrolyte fuel cell (HT-PEFC), which is considered here, GDEs are commonly used. In order to apply the catalyst dispersion onto the GDL, various coating methods can be used such as blade coating, spraying and screen printing. ${ }^{4-6}$ During the subsequent drying process of the dispersion, cracks in the catalyst layer may occur, ${ }^{4,7}$ which segment the catalyst layer into parts of a few hundred micrometers extension. The cracks, which are clearly visible, are not electrochemically active. A review about the formation and analysis of the crack structure as well as an ex-situ analysis of the crack width distribution in GDEs of HT-PEFCs was given by Froning et al. ${ }^{8,9}$ However, it is not yet clear how the crack structures within the catalyst layers affect the performance of the fuel cell. Furthermore, the influence of the morphology of the active catalyst area on performance is not well understood neither. Therefore, the aim of this paper consists in the study of structure-activity relationships by application of complementing ex-situ and in-situ methods. We also aim for understanding the relationship between local morphologies and local activity on one side and cell performance on the other side. Such an understanding together with appropriate measurement technologies would provide a mean of local functional testing of GDE after their processing and before there further assembly to fuel cells after which performance tests are possible only on the cell level. Ideally such testing could be integrated as an online monitoring tool. However, such an endeavor requires identification of methods that allow for the elucidation of structure-activity relationships.

\footnotetext{
*Electrochemical Society Member.

${ }^{\text {z} E-m a i l: ~ g u n t h e r . w i t t s t o c k @ u n i-o l d e n b u r g . d e ; ~ w . l e h n e r t @ f z-j u e l i c h . d e ~}$
}

Scanning electrochemical microscopy (SECM) is an imaging technique in which a microelectrode (ME) is scanned across an immersed sample while recording the current response, ${ }^{10,11}$ which depends on both the electrochemical activity and surface topography of the substrate. ${ }^{12}$ This technique represents one possible method for the determination of the local electrochemical activity of technical electrodes although the number of reported SECM studies on technical electrodes has remained moderate due to difficulties in dealing with rough samples and challenging process conditions (e.g. evolution of gas bubbles, media composition, temperature and pressure conditions). ${ }^{13-16}$ Among the different working modes, the redox competition (RC) mode of SECM uses the competition of the ME and the sample for the same reagent (here dissolved dioxygen, $\mathrm{O}_{2}$ ). ${ }^{17}$ This mode can be used to assess surface and bulk activities of GDEs. In PEFCs, the energy efficiency is mainly limited by the oxygen reduction reaction (ORR) on the cathode because of its sluggish kinetics. ${ }^{18,19}$ During the SECM experiment, the ME is polarized at a fixed potential for diffusion-controlled ORR to occur at the ME, while a suitable potential program is applied to the GDE sample. Oxygen reduction proceeds on the GDE sample and changes the local $\mathrm{O}_{2}$ concentration close to the GDE. This leads to a decrease in the ME current. Thus, high local electrocatalytic activity of the GDE is reflected by low ORR currents at the SECM ME. The intermittent ORR imposed by the potential program on the GDE is necessary to avoid $\mathrm{O}_{2}$ depletion in a macroscopic diffusion layer.

In this paper, two different GDEs were prepared by using poly(tetrafluoroethylene) (PTFE) of different particle sizes as binder. The morphological properties of the resulting two GDEs were measured and compared. Then shear force-based SECM was employed to determine the activity of technical electrodes. Finally we examined these two kinds of GDEs by performing single HT-PEFC measurements.

\section{Experimental}

Preparation of GDEs and single cell test.-A detailed description of the GDE preparation as well as of the MEA assembly can be found in Ref. 3. The GDEs were produced by a doctor blade technique. $20 \%$ $\mathrm{Pt} / \mathrm{C}$ powder (HISPEC 3000) were mixed with deionized water and 
solvent (Propan-1-ol:Propan-2-ol (1:1)) and sonicated for 4 min. Then PTFE was added to the ink and sonicated for another $30 \mathrm{~min}$. After the sonication step, the catalyst ink was coated on a commercially available GDL composed of carbon non-woven with a microporous layer on one side (Freudenberg H2315C2). Finally the electrode was dried in air overnight before use. The final platinum loading for all electrodes was $(0.95 \pm 0.05) \mathrm{mg} / \mathrm{cm}^{2}$ and the PTFE binder loading in the catalyst layer was fixed at $40 \mathrm{wt}-\%$. Two different types of electrodes were produced for the analysis of the electrochemical performance differing in the size of PTFE particles (3M Dyneon, particle sizes of 5060Z and 5032Z PFEE are 220 and $160 \mu \mathrm{m}$, respectively). The GDEs were examined by scanning electron microscope (SEM) coupled with a backscattered electron detector and energy dispersive X-ray (EDX) mapping using a Zeiss Gemini Ultra Plus microscope.

For the single cell testing of the electrodes, MEAs were prepared and equipped with an ABPBI membrane (poly[2,5-benzimidazole], FuMA-Tech, Bietigheim-Bissingen, Germany, doping level $=470$ mass- $\%$, thickness of doped membrane $=85 \mu \mathrm{m})$. The active area for all electrodes was $17.64 \mathrm{~cm}^{2}$. A serpentine five meander-shaped flow field with channel geometries of width $\times$ height $=1 \mathrm{~mm} \times$ $1 \mathrm{~mm}$ was used for anode and cathode sides. A $72 \mathrm{~h}$ break-in procedure was conducted on these MEAs by using a stoichiometry of $2 / 2$ and a current density of $200 \mathrm{~mA} / \mathrm{cm}^{2}$. Afterwards, the polarization curves were attained by measuring the cell voltage while incrementing the current stepwise by $0.88 \mathrm{~A}$ at a constant operating temperature of $160^{\circ} \mathrm{C}$. The used gases were not humidified, and both cells were operated with no back pressure.

Microelectrode preparation.-The initial steps of ME preparation followed guidelines from literature. ${ }^{20,21}$ Specifically, a quartz capillary (OD $0.9 \mathrm{~mm}$, ID $0.3 \mathrm{~mm}$, length $100 \mathrm{~mm}$, Hilgenberg GmbH, Malsfeld) was cut to a length of $9 \mathrm{~cm}$, rinsed once with $10 \% \mathrm{HNO}_{3}$ and several times with deionized water and dried at $100^{\circ} \mathrm{C}$ overnight. A clean Pt wire (25 $\mu \mathrm{m}$ diameter, $99.99 \%$, glowed, Goodfellow $\mathrm{GmbH}$, Bad Nauheim, Germany) was inserted into the middle part of the capillary. A laser-based micropipette puller (P-2000, Sutter Instrument, Novato, CA, USA) was employed to seal the wire into the capillary using 12 cycles of $20 \mathrm{~s}$ heating (puller settings: HEAT 640 FIL 4 VEL 100 DEL 100 PUL 0) and 20 s cooling. During the process, vacuum was applied from both sides of the capillary using a rotary vacuum pump (Vacuubrand $\mathrm{GmbH} \& \mathrm{Co} \mathrm{KG}$, Wertheim, Germany). After sealing, a hard pull was conducted (HEAT 710 FIL 2 VEL 100 DEL 100 PUL 220). The resulting thin tip was polished in a vertical position to a diameter of less than $2 \mu \mathrm{m}$ using a microgrinder (EG-400, Narishige, Tokyo, Japan). After determination of the overall probe dimensions by confocal laser scanning microscopy (CLSM) in the reflection mode, the probe was polished at an angle of 30 degree with respect to the surface normal to give an oval tip with the active area centrically embedded in a plane inclined to the axis of the wire (Figure 3). The effective radius $r_{\mathrm{T}}$ of the electrode after polishing was determined as $\sim 0.9 \mu \mathrm{m}$ by cyclic voltammetry using the relation for a steady-state current for reduction of $4 \mathrm{mM}\left[\mathrm{Ru}\left(\mathrm{NH}_{3}\right)_{6}\right]^{3+}$ in $0.1 \mathrm{M}$ $\mathrm{KCl}$ with an diffusion coefficient of $D=7.4 \times 10^{-6} \mathrm{~cm}^{2} \mathrm{~s}^{-1} .22$ The final dimensions of the inclined electrode were again ascertained by CLSM.

SECM setup.-The SECM setup consists of a three-dimensional positioning system (Digi-Mic two-phase stepper motors with PCSM32 control board, Owis GmbH, Staufen; Germany) and a bipotentiostat ( $\mu$ Bip2, M. Schramm, University of Düsseldorf, Germany), interfaced to a personnel computer (PC-I) via an AD/DAboard (AD/DA board, PCI DAS1602/16, Plug-in Electronic GmbH, Eichenau, Germany). The system was operated under the in house software SECMx. ${ }^{23}$ The horizontal vibration of the ME for shear force (SF) mode was excited and detected by two piezo electric plates (Piezomechanik Pickelmann GmbH, Munich, Germany) glued to home-made brass holders for fixation. ${ }^{24}$ The brass holders were twisted by $45^{\circ}$ to each other. ${ }^{24}$ A DS4L-controller with internal lockin amplifier LIA and SXM control software version 0.19f (Anfatec

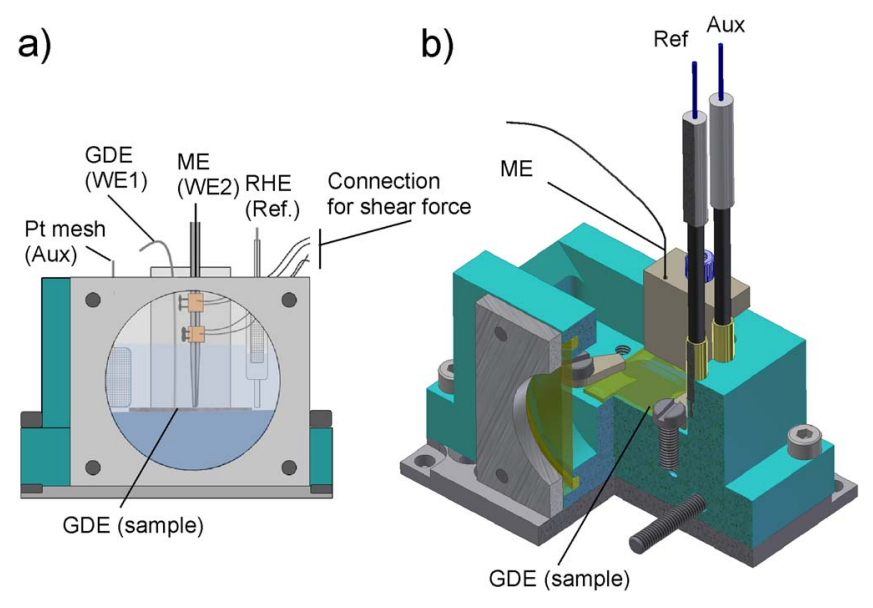

Figure 1. a) Front view and b) side view of the used home-made SECM cell.

Instrument AG, Oelsnitz, Germany) was used to excite and detect the SF signals and to provide the positional feedback to a piezoelectrical actuator (P-625.1CD and controller E-665.CR, Physikinstrumente, Karlsruhe, Germany). An independent personnel computer (PC-II) was used to run the SXM software. ${ }^{25}$ The positional information for the DS4L controller was also recorded by the AD/DA card in PC-I. This information was saved as vertical position information.

The measurements were conducted in a home-made SECM cell equipped with an optical window. In this cell, the sample was fixed by Teflon holders and contacted by a pierced Teflon finger (Figure 1). The GDE was fixed at the bottom of the SECM cell with one Teflon holder and connected with a second Teflon arm holding a Pt wire. A window made of quartz glass allowed the visual observation of the ME. $50 \mathrm{mM} \mathrm{H}_{2} \mathrm{SO}_{4}$ was used as electrolyte and a slight $\mathrm{O}_{2}$ flow was passed above the solution during the measurement. A reversible hydrogen electrode (RHE) was used as reference electrode (RE). A Pt mesh served as auxiliary electrode (Aux).

SECM measurements.-The 4D-SF/SECM measurements were performed in constant distance mode using a SF control. A new routine in SECMx ('4D-Experiment') was used to measure a single cyclic voltammogram $(\mathrm{CV})$ of the sample after each translation step while the $x$ - and $y$-motors were resting. ${ }^{26-29}$ The CVs were performed after a quite time of $2 \mathrm{~s}$ between $0.8 \mathrm{~V}$ and $0.1 \mathrm{~V}$ at a scan rate $v=0.2 \mathrm{~V} / \mathrm{s}$. During the execution of the CVs, the ME potential $E_{\mathrm{T}}$ was held at $0.4 \mathrm{~V}$ vs. RHE and the resulting currents at the ME $\left(i_{\mathrm{T}}\right)$ and the substrate $\left(i_{\mathrm{S}}\right)$ were recorded. The experiments yielded two 4-dimensional data sets $\left(i_{\mathrm{T}}=f\left(x_{m n}, y_{m n}, E_{\mathrm{S}}(t)\right)\right.$ and $i_{\mathrm{S}}=f\left(x_{m n}, y_{m n}, E_{\mathrm{S}}(t)\right)$ and one 3-dimensional data set $\left(z=f\left(x_{m n}, y_{m n}\right)\right.$ that were processed off-line using an expanded version of the in house software MIRA. ${ }^{30}$ For brevity we abbreviate this procedure as 4D-SF/SECM (for a discussion with respect to literature see 4D-SF/SECM imaging section).

\section{Results and Discussion}

SEM and EDX mapping of GDEs.-Two GDE samples were prepared by the doctor blade method using different PTFE binders. Figure 2 shows the SEM and EDX mappings of the GDEs obtained with PTFE particle size of $220 \mu \mathrm{m}$ (GDE 1) and $160 \mu \mathrm{m}$ (GDE 2). Clearly, both electrodes show cracks caused by isopropanol evaporation during the drying process. As can be seen from a comparison of the SEM backscattered electron images, the surface morphology varies with the PTFE binder used despite the same mass content of PTFE. The GDE1 made from larger PTFE particles show cracks with strongly changing width while GDE 2 has more uniform width of the cracks. In addition, various gray levels can be identified in the SEM images. There are larger light gray areas in the image of GDE1. EDX mapping was used to further study the material distribution in 

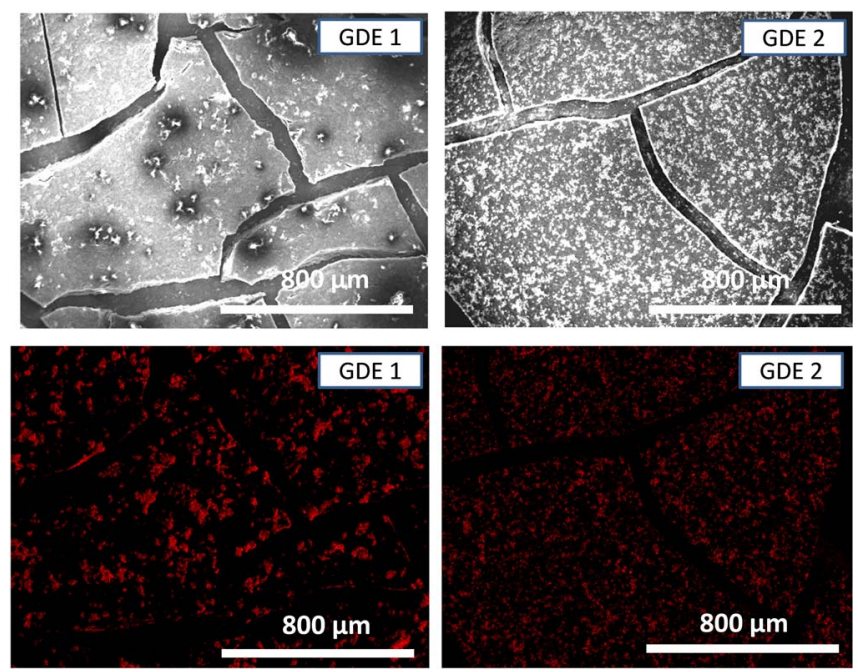

Figure 2. SEM backscattered electrons (top) and EDX mapping (bottom) of fluorine $\mathrm{K}_{\alpha}$ emission of GDE 1 and GDE 2 produced from PTFE particle sizes of 220 and $160 \mu \mathrm{m}$, respectively.

both GDEs (Figure 2). Fluorine is a constituent only of the PTFE and therefore the fluorine distribution indicates the distribution of PTFE in the composite electrode. It is obvious that PTFE particles are more uniformly dispersed and show less agglomerates on the surface of GDE2 for which the smaller PTFE particles were used. Boths GDEs were used for the analysis of the electrochemical performance in the following parts.

ME properties.-The 4D-SF/SECM experiments were conducted employing an inclined ME in order to facilitate the diffusion of oxygen into the gap between the probe and the sample during the resting period of $2 \mathrm{~s}$. This is necessary because otherwise a rather active sample would always deplete the $\mathrm{O}_{2}$ content resulting in a ME ORR current close to zero. This would prevent a differentiation between two rather active electrodes. Figure 3 shows a microphotograph and schematic representation of the employed ME. The average distance $d$ between the active electrode area and a plane surface was calculated to be $\sim 5 \mu \mathrm{m}$ utilizing the known probe dimensions and additionally confirmed by SECM current-distance curves recorded when retracting the electrode away from a glass sample. Inclined disk microdisk electrodes have been used before for combined topographical and electrochemical imaging because it provides a smaller and more defined area for mechanical interaction and thus higher lateral resolution in topographic imaging as an additional advantage. ${ }^{31-33}$ A similar principle is also used in soft microelectrode arrays. ${ }^{34}$ In all those cases the distance between the active electrode area of the ME and the sample surface is defined by the shape of the probe. a)

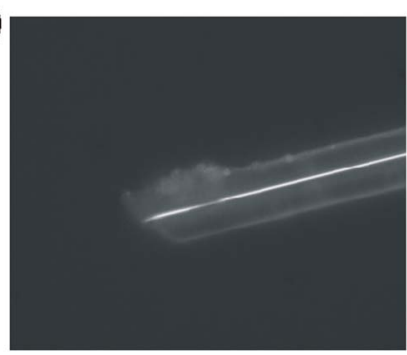

b)

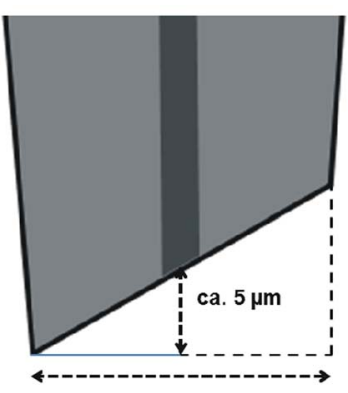

ca. $17 \mu \mathrm{m}$
Figure 3. a) Microphotograph and b) schematic representation of the ME dimensions. a)

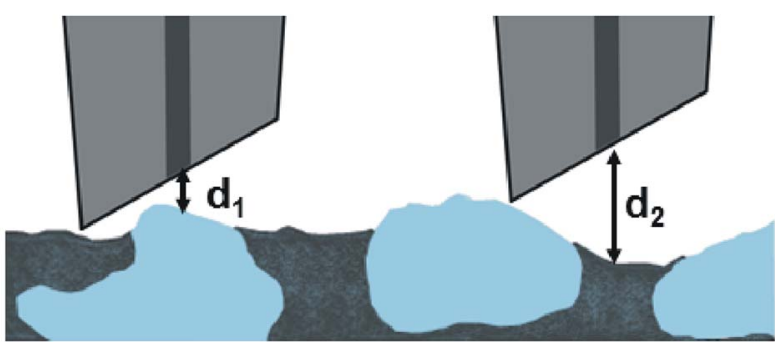

b)

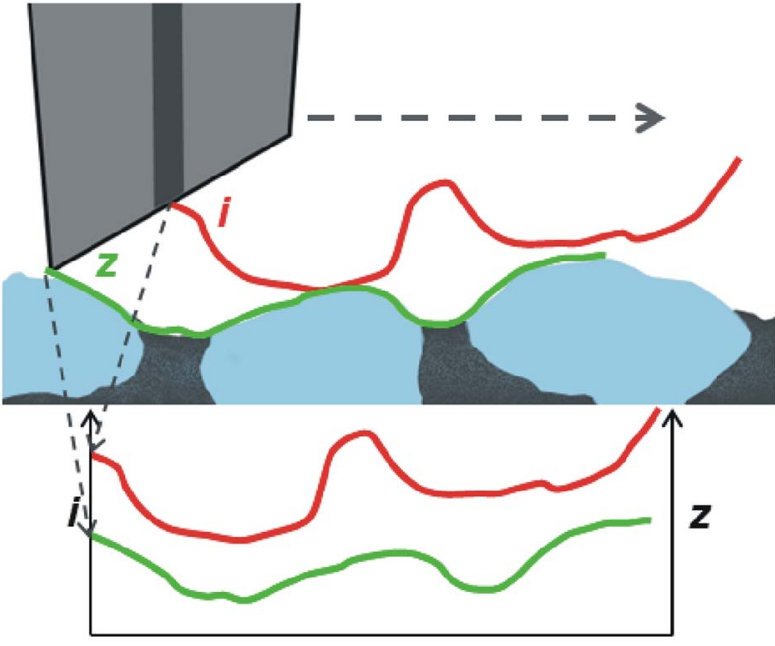

$\mathbf{X}$

Figure 4. Influence of the lateral distance between the mechanic contact point of the glass sheath and the active area of the inclined ME.

Due to the constrains of the smallest distance between the active ME area and the sample by the shape of the ME, the SECM data of the inclined ME differs from that of conventional microdisk electrode typically described as a leaky thin-layer cell. ${ }^{11}$ Those differences affect for instance the feedback current in an approach curve. An inclined ME does not reach the contrast expected from a parallel orientation of sample and ME due to a inefficient shielding of the diffusion from the solution bulk. ${ }^{31,33}$ Furthermore, the diffusion of redoxactive species is unsymmetrically blocked close to the sample surface ${ }^{35}$ while no significant deviations are expected in the bulk solution. During the imaging process in constant distance mode, the lateral distance between active ME area (for activity sensing) and the mechanical point of contact between the glass sheath and the sample surface (i.e. the reference point for the shear force distance control) might cause finite probe size effects by slightly different working distances for the electrochemical measurement. ${ }^{33,36}$ This applies to topographically inhomogeneous samples as indicated in Figure 4a. Additionally, there is an offset of $8.5 \mu \mathrm{m}$ (Figure 3) between the electrochemical and topographical data as illustrated in Figure 4b. This corresponds to about 1.5 times the selected smaller step size $\Delta x=5 \mu \mathrm{m}$ in the reported images.

4D-SF/SECM imaging.-Pt nanoparticles on carbon are very efficient oxygen reduction catalysts. Therefore, it is very difficult to differentiate between slightly varying activities by redox competition (RC) mode imaging using coplanar oriented MEs even with intermittent oxygen generation pulses, ${ }^{17}$ because the $\mathrm{O}_{2}$ will be depleted rapidly in the gap between the oxygen reducing sample and the ME. The inclined ME applied here does not only afford higher resolution in topography imaging but also counteracts the depletion of oxygen in the gap between the sample surface and the ME. In addition, oxygen depletion was also mitigated by probing the oxygen reduction during a sample $\mathrm{CV}$ between $0.8 \mathrm{~V}$ to $0.1 \mathrm{~V}$ at every grid point of the 


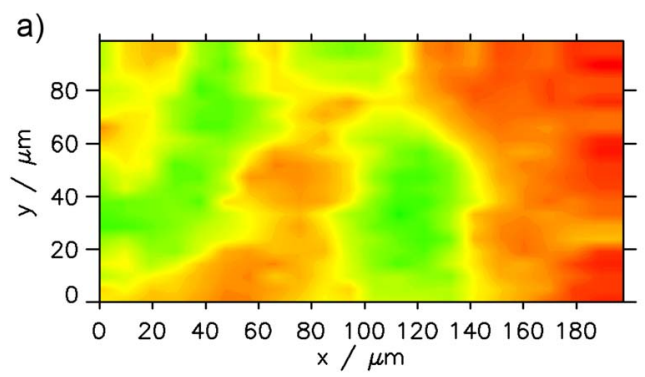

b)

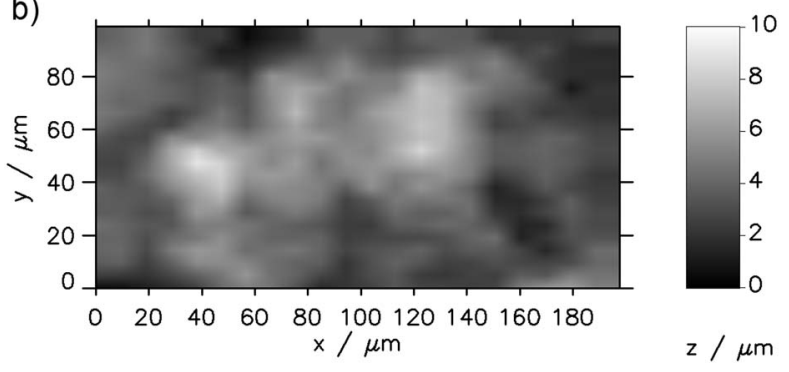

Figure 5. 4D-SF/SECM image of GDE 1; a) electrochemical data, b) topography. electrolyte was $50 \mathrm{mM} \mathrm{H}_{2} \mathrm{SO}_{4}$; experimental parameters of the sample $\mathrm{CV}$ at every grid point $E_{\text {start }}=0.8 \mathrm{~V}, E_{\text {low }}=0.1 \mathrm{~V}, E_{\text {end }}=0.8 \mathrm{~V}, v=0.2$ $\mathrm{V} / \mathrm{s}, E_{\mathrm{T}}=0.4 \mathrm{~V}$; Imaging parameters $\Delta x=5 \mu \mathrm{m}, \Delta y=10 \mu \mathrm{m}$. Spikes were removed from the data (see text for details and discussion).

image. ${ }^{27}$ The term "4D Shearforce-Based Constant-Distance Mode" has been used before by Nebel et al.$^{37}$ for SECM experiments in which the ME recorded a current distance curve at each grid point. The authors demonstrated reconstruction of three-dimensional concentration distributions around microelectrodes of round $50 \mu \mathrm{m}$ diameter from the dataset $\left(x, y, z, i_{\mathrm{T}}\right){ }^{37}$ For brevity we call our experiments 4DSF/SECM. However, we would like to point out that our experiments yield different types of data namely two four-dimensional dataset ( $x$, $\left.y, E_{\mathrm{S}}(t), i_{\mathrm{T}}\right)$ and $\left(x, y, E_{\mathrm{S}}(t), i_{\mathrm{S}}\right)$. The potential scan started at values where no oxygen reduction occurs which allowed a periodic re-supply of oxygen to the GDE between probing ORR during the negative going scan of each CV. The ME was held at a constant reduction potential of $0.4 \mathrm{~V}$ vs. RHE at which the diffusion-controlled ORR occurs. If the sample potential reaches a value at which the ORR proceeds at the sample as well, GDE and ME compete for $\mathrm{O}_{2}$ (RC mode).

An additional three-dimensional data set $(x, y, I)$ shown in the false-color image were extracted from the ME currents $i_{\mathrm{T}}(0.4 \mathrm{~V})$ recorded when the sample potential $E_{\mathrm{S}}$ was $0.4 \mathrm{~V}$ (vs. Pt reference electrode) in the negative going scan. This value was normalized by the diffusion-limited ME current $i_{\mathrm{T}, \infty}$ recorded for the ORR in the bulk phase (Equation 1).

$$
I=i_{\mathrm{T}}(0.4 \mathrm{~V}) / i_{\mathrm{T}, \infty}
$$

The relative current $I$ indicates the decrease of the steady state ORR current at the ME if it is located close to the sample and the sample also reduced oxygen thus competing for the same reagent as the ME.

Figure 5 shows the activity map (a) and the topography (b) of GDE1. Spikes were removed from the original data by the software MIRA. ${ }^{30}$ A data point is considered a spike when the current difference to the neighboring location is larger than physically possible with a ME of a given radius. This applied to 49 data points. The origin of these spikes is not clear and varies between experiments. Compared to conventional SECM measurements, we have to use lower time constants for the 4D-SF/SECM measurements as the sample potential is scanned. Additionally, we noted that samples with very inhomogeneous micromechanical properties (such as composites with larger polymer parts) and large corrugation tend to produce more spikes. This can be related to the difficulty to maintain a constant working distance in a shear force regime if topographical roughness
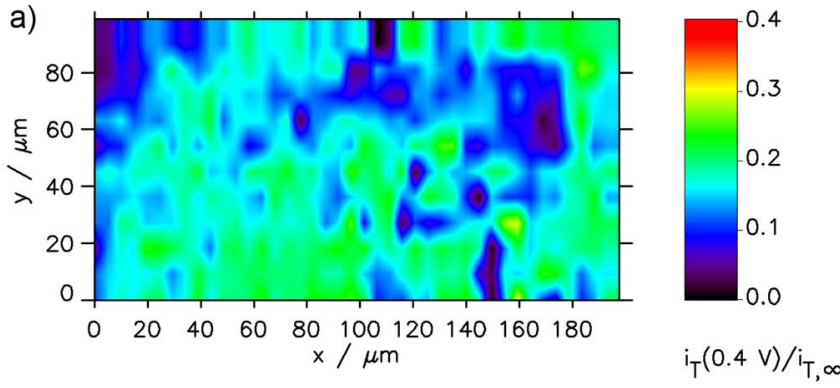

b)
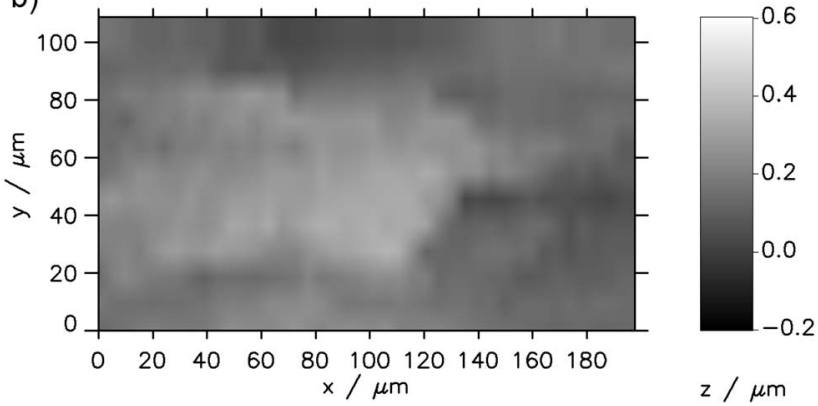

Figure 6. 4D-SF/SECM image of GDE 2; a) electrochemical data, b) topography; electrolyte was $50 \mathrm{mM} \mathrm{H}_{2} \mathrm{SO}_{4}$; experimental parameters of the sample $\mathrm{CV}$ at every grid point $E_{\text {start }}=0.8 \mathrm{~V}, E_{\text {low }}=0.1 \mathrm{~V}, E_{\text {end }}=0.8 \mathrm{~V}, v=0.2 \mathrm{~V} / \mathrm{s}$, $E_{\mathrm{T}}=0.4 \mathrm{~V}$; Imaging parameters $\Delta x=5 \mu \mathrm{m}, \Delta y=10 \mu \mathrm{m}$.

and material contrast occur. Relating shear force signals purely to a distance-dependence is an approximation that holds for samples with homogeneous distribution of stiffness. However, when this property varies, the shear force signal also reacts to (mechanical) material contrast and may cause intermediate contacts of the probe and the sample especially if the sample topography changes strongly. Such disturbances may show up in the current signal as spikes during an intermediate contact despite the fact that the distance variation of a few hundred nanometer is still very small compared to the working distance of our probes (Figure 3). Despite the spikes, the SF regulation helps to accommodate the large topographic features of up to 10 $\mu \mathrm{m}$ in (Figure 5a). GDE1 shows an inhomogeneous current distribution over areas of several ten micrometers (Figure 5a) with relative currents $I$ between 0.2 and 0.4 . This means that the oxygen has not been completely consumed by the substrate leaving some $\mathrm{O}_{2}$ to be reduced at the ME. This observation can be explained by a limited substrate activity. The topographical data also shows a heterogeneous height profile. Most likely, these heterogeneities are caused by the PTFE agglomerates which are already visible within the SEM image of the sample (Figure 2). The shift between the electrochemical map and the topography image, as discussed in the context of Figure 4, is relatively small with respect to the ME dimensions and the step size of $5 \mu \mathrm{m}$. Despite intensive efforts to determine correlations between the electrochemical activity and the height profile, no direct correlation was found. Consequently, topographical effects cannot necessarily be associated with inactive areas like Teflon agglomerates.

Contrarily, GDE2 (Figure 6) shows only minor inhomogeneities of $I$, but a considerably decreased currents at the $\mathrm{ME}(0.0<I<0.2$, Figure 6a). This reflects the overall higher activity of this GDE2. In agreement with the homogeneous appearance of the substrate, the topography map (Figure 6b) shows height variations below $1 \mu \mathrm{m}$. Since both types of GDEs were characterized with the same inclined $\mathrm{ME}$, the oxygen diffusion field during the scan is comparable for both samples. According to the SECM results, GDE2 shows a more homogeneous morphology and higher electrochemical activity for ORR than GDE1.

The SECM investigation for this in this proof of principle study were conducted in $50 \mathrm{mM} \mathrm{H} \mathrm{SO}_{4}$ as electrolyte. This electrolyte system works stable at room temperature and many data exist for 


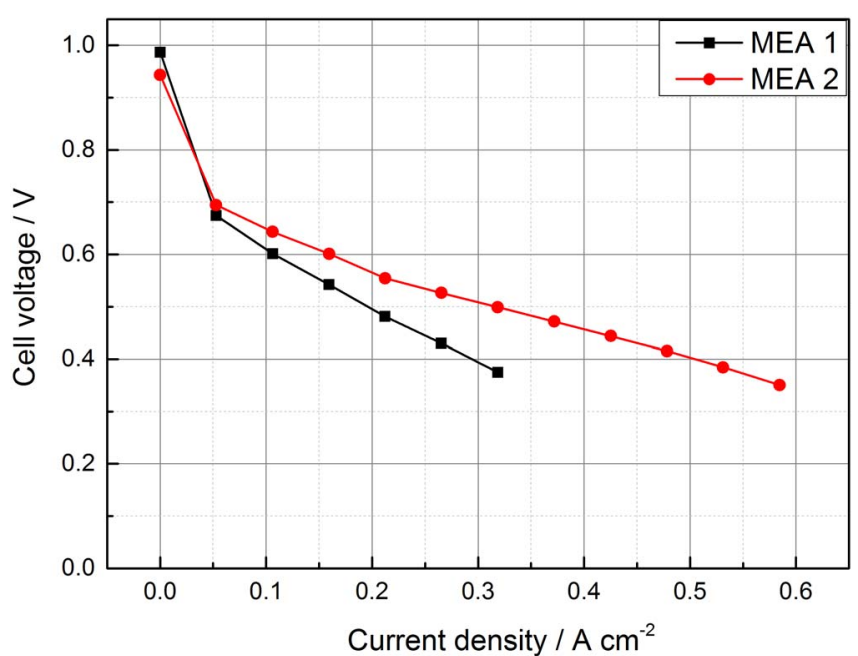

Figure 7. $\mathrm{H}_{2}$-air HT-PEFC performance of ABPBI-based MEAs using GDE 1 and GDE 2 after $72 \mathrm{~h}$ break-in procedure, operated at $160^{\circ} \mathrm{C}$, ambient pressure and $\lambda=2 / 2$.

comparison. We expect that SECM measurements allow an assessment of the catalytic activity of the Pt catalyst after the productions steps for the GDE and should show the influence of aggregation within the complex composite material of the GDE and be predictive for one aspect of the GDE performance in fuel cell tests. The decision for $\mathrm{H}_{2} \mathrm{SO}_{4}$ as electrolyte for the SECM experiments (rather than diluted $\mathrm{H}_{3} \mathrm{PO}_{4}$ ) avoided possible complication with the adsorption of phosphate at $\mathrm{Pt}$ (at the sample and at the ME probe) at room temperature.

Polarization curves.-In order to observe the correlation between the morphology of the different GDEs (depicted in the SEM and SF-SECM results) and local ORR activity (from RC-SECM data) on one side and the fuel cell performance on the other side, MEA1 and MEA2 were made from GDE1 and GDE2. Both MEAs were then evaluated in a HT-PEFC test station at $160^{\circ} \mathrm{C}$ with dry gases in co-flow mode and a stoichiometry of two on both sides. The testing results in Figure 7 are in line with the RC-SECM activity data. MEA2 shows better performance than MEA1 over the whole range of current densities. The slightly higher cell voltage of MEA2 at $0.05 \mathrm{~A} \mathrm{~cm}^{-2}$ seems to be caused by the better catalytic activity toward ORR of GDE2. At a current density of $0.2 \mathrm{~A} / \mathrm{cm}^{2}$, the voltages of MEA1 and MEA2 are $0.504 \mathrm{~V}$ and $0.559 \mathrm{~V}$, respectively. As current density increases, the cell with MEA1 interestingly shows a steeper drop of the cell voltage than the cell with MEA2 which implies a higher ohmic resistance of this cell compared to cell with MEA2. A major part of the ohmic resistance is related to the ionic transport inside the membrane. In order to avoid differences in the cell performance due the used membrane, both membranes had been cut from the same ABPBI sample. The resulting differences in the cell performances can therefore be related solely to the differences in conductivity of the catalyst layers. It was shown previously that the phosphoric acid distribution as well as the composition of the phosphoric acid plays a major role in the fuel cell behavior. ${ }^{38,39}$ In order to explain these effects in more detail, further experiments e.g. in operando observation of the phosphoric acid distribution inside a running cell will be necessary to obtain additional information of the processes leading to different conductivities.

\section{Conclusions}

Two GDEs made with PTFE binders of different particle sizes were characterized by SEM and tested in $\mathrm{H}_{2}$ /air single HT-PEFCs. The morphologic analysis demonstrated that the GDE made with smaller PTFE particles exhibited more regular cracks and a smoother surface, possessed better electrochemical performance in polarization curves of HT-PEFCs. SF-SECM in the RC mode was used to assess simultaneously morphology and electrochemical activity. The use of inclined ME avoided the complete depletion of dissolved $\mathrm{O}_{2}$ close to the GDEs and allowed a differentiation between both GDEs. The GDE with smaller PTFE particles showed higher catalytic activity toward ORR, more homogeneous current distribution and morphological appearance. These findings from SECM measurements agree well with morphologic characterization by SEM and HT-PEFC performance tests. Therefore, SECM might be used as a promising tool to evaluate the catalytic quality of GDEs before applying them to HT-PEFCs despite the fact that SECM and HT-PEEC tests are conducted under different temperature and different acids. An ultimate objective would be the development of an online monitoring method integrated into a coating system. The instrumentally demanding SF system could then be replaced by flexible SECM probes ${ }^{34,35,40,41}$ that also would allow the integration of microfluidic components for only locally wetting a sample GDE. ${ }^{42}$

\section{Acknowledgments}

The work was funded by the State of Lower Saxony and the EWE Foundation within the PhD program "System Integration of Renewable Energies" and by the State of Lower Saxony and the VW Foundation (ZN 2783) within the PhD program "Energy Storage and Electromobility Lower Saxony (GEENI)". Part of this work was supported by funding from the China Scholarship Council (No. 201506890023)

\section{References}

1. B. Emonts, S. Schiebahn, K. Görner, D. Lindenberger, P. Markewitz, F. Merten, and D. Stolten, J. Power Sources, 342, 320 (2017).

2. M. Carmo, D. L. Fritz, J. Mergel, and D. Stolten, Int. J. Hydrogen Energy, 38, 4901 (2013).

3. F. Liu, S. Mohajeri, Y. Di, K. Wippermann, and W. Lehnert, Fuel Cells (Weinheim, Germany), 14, 750 (2014)

4. F. Mack, M. Klages, J. Scholta, L. Joerissen, T. Morawietz, R. Hiesgen, D. Kramer, and R. Zeis, J. Power Sources, 255, 431 (2014).

5. T. Shu, D. Dang, D.-w. Xu, R. Chen, S.-j. Liao, C.-t. Hsieh, A. Su, H.-y. Song, and L. Du, Electrochim. Acta, 177, 168 (2015).

6. H. Su, T.-C. Jao, O. Barron, B. G. Pollet, and S. Pasupathi, J. Power Sources, $\mathbf{2 6 7}$ 155 (2014).

7. M. Kim, G. Jeong, K. Eom, E. Cho, J. Ryu, H.-J. Kim, and H. Kwon, Int. J. Hydrogen Energy, 38, 12335 (2013).

8. D. Froning, W. Maier, J. Gross, T. Arlt, I. Manke, and W. Lehnert, Int. J. Hydrogen Energy, 39, 9447 (2014).

9. D. Froning, W. Maier, J. Groß, T. Arlt, I. Manke, and W. Lehnert, International Journal of Hydrogen Energy, 39, 9447 (2014).

10. A. J. Bard and M. V. Mirkin (Ed.), Book Scanning Electrochemical Microscopy, CRC Press Taylor \& Francis Group, Boca Raton, London, New York (2012).

11. G. Wittstock, M. Burchardt, S. E. Pust, Y. Shen, and C. Zhao, Angew. Chem. Int. Ed., 46, 1584 (2007)

12. J. Kim, C. Renault, N. Nioradze, N. Arroyo-Curras, K. C. Leonard, and A. J. Bard, J. Am. Chem. Soc., 138, 8560 (2016).

13. S. Schmachtel, S. E. Pust, K. Kontturi, O. Forsen, and G. Wittstock, J. Appl. Electrochem., 40, 581 (2010).

14. H. Bülter, F. Peters, J. Schwenzel, and G. Wittstock, Angew. Chem. Int. Ed., 53, 10531 (2014).

15. A. R. Zeradjanin, N. Menzel, W. Schuhmann, and P. Strasser, Phys. Chem. Chem. Phys., 16, 13741 (2014).

16. A. R. Zeradjanin, T. Schilling, S. Seisel, M. Bron, and W. Schuhmann, Anal. Chem., 83, 7645 (2011).

17. K. Eckhard, X. Chen, F. Turcu, and W. Schuhmann, Phys. Chem. Chem. Phys., 8, 5359 (2006).

18. I. Katsounaros, S. Cherevko, A. R. Zeradjanin, and K. J. J. Mayrhofer, Angew. Chem Int. Ed., 53, 102 (2014).

19. Y. -J. Deng, G. K. H. Wiberg, A. Zana, and M. Arenz, Electrochim. Acta, 204, 78 (2016).

20. B. B. Katemann and W. Schuhmann, Electroanalysis, 14, 22 (2002).

21. M. A. Mezour, M. Morin, and J. Mauzeroll, Anal. Chem., 83, 2378 (2011).

22. J. Kim and A. J. Bard, J. Am. Chem. Soc., 138, 975 (2016).

23. C. N. Kirchner, K. H. Hallmeier, R. Szargan, T. Raschke, C. Radehaus, and G. Wittstock, Electroanalysis, 19, 1023 (2007).

24. B. B. Katemann, A. Schulte, and W. Schuhmann, Chem. Eur. J., 9, 2025 (2003).

25. U. M. Tefashe and G. Wittstock, C. R. Chim., 16, 7 (2013).

26. B. Gründig, G. Wittstock, U. Rüdel, and B. Strehlitz, J. Electroanal. Chem., 395, 143 (1995). 
27. L. Diaz-Ballote, M. Alpuche-Aviles, and D. O. Wipf, J. Electroanal. Chem., 604, 17 (2007).

28. M. Michalak, M. Kurel, J. Jedraszko, D. Toczydlowska, G. Wittstock, M. Opallo, and W. Nogala, Anal. Chem., 87, 11641 (2015).

29. I. Plettenberg and G. Wittstock, Electrochim. Acta, 197, 318 (2016).

30. G. Wittstock, T. Asmus, and T. Wilhelm, Fresenius J. Anal. Chem., 367, 346 (2000).

31. M. Etienne, E. C. Anderson, S. R. Evans, W. Schuhmann, and I. Fritsch, Anal. Chem., 78, 7317 (2006).

32. A. Ueda, O. Niwa, K. Maruyama, Y. Shindo, K. Oka, and K. Suzuki, Angew. Chem. Int. Ed., 46, 8238 (2007).

33. S. E. Pust, M. Salomo, E. Oesterschulze, and G. Wittstock, Nanotechnology, 21, 105709/1 (2010).

34. A. Lesch, D. Momotenko, F. Cortes-Salazar, F. Roelfs, H. H. Girault, and G. Wittstock, Electrochim. Acta, 110, 30 (2013).
35. A. Lesch, B. Vaske, F. Meiners, D. Momotenko, F. Cortes-Salazar, H. H. Girault, and G. Wittstock, Angew. Chem. Int. Ed., 51, 10413 (2012).

36. M. Etienne, J.-P. Moulin, and S. Gourhand, Electrochim. Acta, 110, 16 (2013).

37. M. Nebel, K. Eckhard, T. Erichsen, A. Schulte, and W. Schuhmann, Anal. Chem., 82, $7842(2010)$

38. W. Maier, T. Arlt, K. Wippermann, C. Wannek, I. Manke, W. Lehnert, and D. Stolten, J. Electrochem. Soc., 159, F398 (2012).

39. T. Arlt, M. Wiebke, C. Toetzke, C. Wannek, H. Markoetter, F. Wieder, J. Banhart, W. Lehnert, and I. Manke, J. Power Sources, 246, 290 (2014).

40. A. Lesch, P.-C. Chen, F. Roelfs, C. Dosche, D. Momotenko, F. Cortes-Salazar, H. H. Girault, and G. Wittstock, Anal. Chem., 86, 713 (2014).

41. F. Cortes-Salazar, D. Momotenko, H. H. Girault, A. Lesch, and G. Wittstock, Anal. Chem., 83, 1493 (2011).

42. D. Momotenko, L. Qiao, F. Cortés-Salazar, A. Lesch, G. Wittstock, and H. H. Girault, Anal. Chem., 84, 6630 (2012). 